\title{
Timescales and the Factors Influencing Time Perception
}

\author{
AKI PASOULAS \\ Centre for Music and Audio Technology, University of Kent, Chatham Historic Dockyard, Kent ME4 \\ 4TZ, UK \\ E-mail: a.pasoulas@kent.ac.uk
}

This paper explores timescales within absolute and psychological times, and identifies the many factors that affect our perception of time passing and estimation of durations, which inevitably influence our perception of musical structures; in particular, it discusses listening experiences, and theoretical approaches to psychological states and emotional responses. It proposes a process according to which the time-influencing factors operate between listener and music. The discussion is approached through the lens of the electroacoustic composer and makes references to short excerpts from the author's work and related repertoire. However, as the paper discusses time in relation to sound structures, it is also relevant to other time-based sound art and music.

\section{ABSOLUTE AND PSYCHOLOGICAL TIME}

Time is generally understood as being experienced in two distinct ways. The first is through the notion of absolute or clock time, an objective way of counting mechanically durations via a universal type of measurement that divides time into equal units of fixed proportions, such as the microsecond, the minute, the year or the century. According to this notion, time flows at a constant rate, is independent of events occurring and is the same for every object, animate or inanimate, that exists. 
The second way of experiencing time is based on a concept known as psychological time, by which we understand and experience the world around us, including music. Psychological time flow is elastic; we use expressions like 'time drags' or 'time flies' in order to describe our perception of time passing. Durations can only be estimated or 'felt' when compared with other remembered durations.

\subsection{Physical Timescales}

Absolute time can be divided into several timescales, which I will be referring to as physical timescales, starting and ending with ideal mathematical durations, those of infinity and its opposite of infinitesimality. In between those two extremes we can identify additional scales, such as the seven suggested by Curtis Roads (2001: 3-38). The supra timescale extends beyond the musical composition, into months, decades and larger divisions of time. There are examples of pieces belonging to the supra timescale, such as Jem Finer's LongPlayer, a generative composition designed to play for one thousand years; and John Cage's As Slow As Possible, currently played in Germany on a church organ designed to go on for 639 years. However, we are physically able to perceive only a fraction of those compositions in the more accessible macro scale. According to Roads (ibid.), the macro scale is normally associated with musical form and extends from minutes up to several days in extreme cases. The meso scale comprises the musical phrase and is measured in minutes or seconds; this is not clearly differentiated in terms of duration from Roads' next scale, which is the sound object, the basic unit of musical structure. Evidently, the timescales found in musical pieces are separated because of their function, rather than their measured duration. The sound object scale, which according to Roads can be measured in minutes or seconds, is followed by the micro scale that comprises 
durations extending from the threshold of timbre perception (several hundred microseconds) up to a short sound object. The micro timescale is a special category, since it can transform itself into one single object, or many - an accumulation of single objects that is perceived as a cloud, which becomes a single sound object in itself or a gesture through time. Roads mentions two more timescales, the sample and the subsample, measured in nanoseconds, durations too short to be perceived.

Durations that make sense in music start from the threshold of timbre perception and go up to the macro timescale, which is commonly connected with musical form. Composers sometimes employ timescales that exceed those limits. However, durations less than the micro timescale are musically usable only as mass events and durations that last more than the threshold of human body fatigue can be experienced only in part and not in their entirety.

Even the macro scale, as defined by Curtis Roads (i.e. sometimes extended to several days), may surpass the limits of body fatigue. Composers have found a solution to presenting excessive macro durations to audiences by dividing their lengthy pieces into several sections that can be listened to at intervals. For example, Karlheinz Stockhausen's Licht (Die sieben Tage der Woche) is an epic work divided into seven operas that last in total c. 29 hours. 1 However, in cases where the duration of the piece is not as excessive as in Licht, we should consider that there is one further boundary, an important border after which we cannot use our concentration to fully understand and appreciate musical relationships. In relatively long pieces, some

\footnotetext{
1 Light (The Seven Days of the Week); it was composed between 1977-2002. It can be considered as one piece divided in seven sections, because its structure, based on Stockhausen's concept of 'superformula', stretches to include all seven operas. The 'formula' is Stockhausen's principle of organisation, comprising series of elements at different levels. The opera cycle Licht is planned around three principal formulas, associated with the three main characters Michael, Lucifer and Eve. The three formulas, which have different characteristics, are combined in the superformula, the basic statement of which extends throughout the whole work, so that each opera represents only a section of the superformula (Pasoulas 2001).
} 
people may need to stop listening for a short period of time and then come back to the composition and continue listening, thus dividing the piece into manageable temporal slots. Consequently, long durations that belong to the macro scale cannot be perceived in their entirety. As a result, we need to consider that the macro scale is divided into two parts. The lower macro, which is the timescale that is perceivable in its entirety, it depends on the listener's individual limits of concentration (known as 'attention span'), and may comprise either the whole or part of the musical form; and the upper macro, which extends to the entire duration of musical form, and it may exceed the human capabilities of continuous concentration. Depending on the strength of a person's attention span, there may come a point in time where all or part of the information transmitted by a musical piece is not registered or effectively processed by that person. Since the ability to concentrate varies from person to person, a piece that belongs to the lower macro scale for one listener may belong to the upper macro scale for another.

Because of the subdivision of the macro scale, another problem emerges: the upper macro scale cannot be differentiated from the supra scale, because both comprise long durations that cannot be perceived in their entirety. It becomes apparent that dividing musical information into timescales, in the way Curtis Roads attempts to do, is not a straightforward matter. Timescales can vary, not only according to the duration of a piece of music, a phrase, a gesture, or a sound event, but also according to other factors, such as the function of a sound in a musical passage, or the attention span of individual members of an audience, as explained above.

Ultimately, listeners are only concerned with durations that make sense to them, which comprise the roughly defined scales of lower macro, meso, sound object and micro. The rest of them, i.e. supra, upper macro and the smallest sample and 
subsample, only have a theoretical value for the listener (although they can be used practically by the composer) and remain outside our observational limits.

\subsection{Psychological Timescales}

Sometimes we feel that time passing accelerates and we say that 'time flies', and when the opposite is felt we say that 'time drags'. Two time intervals that have identical actual durations - if measured in absolute time - may be experienced as having entirely different psychological temporal spans (see e.g. Hogan 1978). If we play two sounds of equal actual duration one after the other, it is possible to perceive one of them as longer than the other. When we compare relatively short sounds (a few seconds each), the difference of their psychological temporal spans is negligible, no matter how spectrally different those two sounds are. When comparing contrasting sounds, for example a spectrally fast-changing texture followed by a relatively static texture, the difference between their perceived durations becomes more pronounced when those sounds are long (Sound examples 1 and 2; the first pair of sounds comprises short durations, while the second consists of long sounds; in my experience, usually the more static sound is perceived as longer because it does not have pronounced changes and does not give an indication of when it will end). In cases where long sustained sounds are involved and there is little or no indication of long-term evolution, the listener becomes less aware of time passing. In such cases, time passing can be experienced through our biological clock, our physiological or psychological changes, or through changes in the environment. However, if there are no cues or clues in the sound itself to make us aware of its approximate physical duration, the sound in question appears seemingly endless, or even static. 
In addition to experiencing equal absolute durations as being uneven when sounds play in succession, we can experience diverse psychological durations when equally extended sounds play simultaneously, by directing our attention selectively to various layers of the sound structure. As an example, I will refer to a generative piece of music called Turing 2, composed by Anthony Moore. The version I experienced at Goldsmiths University in 2005 lasted around 50 minutes and consisted of three distinct layers of sounds: the busiest layer comprised short hammer-like sounds; the tempo in that layer was continually changing, but remained within a pre-defined threshold. The second layer had low-frequency long buzzing sounds, and the third layer was almost pink noise-based, continuous, mimicking the sound of a ventilator. The piece evolved slowly, until it reached a point of a rather vigorous activity of the short sounds, which signalled a return to the original state. Three returns to the initial state were the only indication of time passing, although it was not apparent whether they were equally spaced within the structure of the piece. After listening for a while, I could not tell whether the piece was in its 25 th or 40 th or 50 th minute. Additionally, when I alternated my focus among the three layers, I had different experiences of time passing and perceived durations. Concentrating on the layer with the hammer-like sounds, which had more events in a time interval compared to the rest, gave me the feeling of a quicker pace of music than when focusing on the second layer with the long buzzing sounds, which had fewer changes; whereas directing my attention to the continuous ventilator sounds gave me the impression of near stasis. Naturally, my brain was constantly comparing the layer on which I was concentrating with the rest of the layers that were still there, although in the background of my attention. Because of this subconscious comparison, a layer would seem to run faster or slower when matched against its neighbouring layers; in addition, a time interval with more 
changes seemed shorter than an interval with fewer changes with comparable physical duration. This observation agrees with that by psychologist Wayne Hogan, who concluded that intervals with moderately complex stimuli are experienced shorter than those with minimal information (1978: 423; see also discussion in Pasoulas 2011a).

I perceived three concurrent layers of identical actual temporal span, which each nevertheless gave a different impression of duration that changed with the focus of attention. The piece that I used as an example gave me the opportunity to make a few additional observations. First, a timescale dominates when the listener pays attention to it; and second, a timescale attracts the listener's attention if it contains important sonic material, although we cannot predetermine which characteristics sonic material must have to be important, as that depends on context. However, an important question remained: was the comparison among the layers the only factor that changed my time perception?

\section{THE PSYCHOLOGICAL TIME CONTINUUM}

Before delving into the above question regarding factors that affect time perception, we need to understand what makes us perceive a single sound as being long or short, and also whether there is any connection between the perceived feeling and the actual duration. When talking about psychological durations, I am tempted to substitute the word 'feeling' for the word 'duration'. The reason is that when we discuss psychological time, it is not possible to use a metric system (e.g. two minutes, or ten minutes long). We substitute a qualitative for a quantitative model when we express our feelings about perceived durations and time passing (e.g. it feels long; it is hasty; it is monotonous). Hence, I prefer to use the terms 'haste' and 'languor', thus 
replacing words such as 'short' and 'long'. 'Languor' refers to a feeling of lack of interest or energy, which may lead to boredom; it also shows inactivity and a relaxed and comfortable state.

Consequently, instead of dividing psychological time into a number of timescales, I propose a continuum as shown in Fig. 1, where haste and languor occupy the opposite poles. The listener subconsciously selects different positions on this continuum, resulting in differing perceptions of time passing and interpretations of actual durations. At the exact centre of the continuum there is balance, where actual (measured) duration is supposed to equal psychological (perceived) duration, as neither haste nor languor is experienced. When the selection point tends towards languor, the listener perceives a time interval as being increasingly longer than it actually is; the opposite occurs when the selection point tends towards haste.

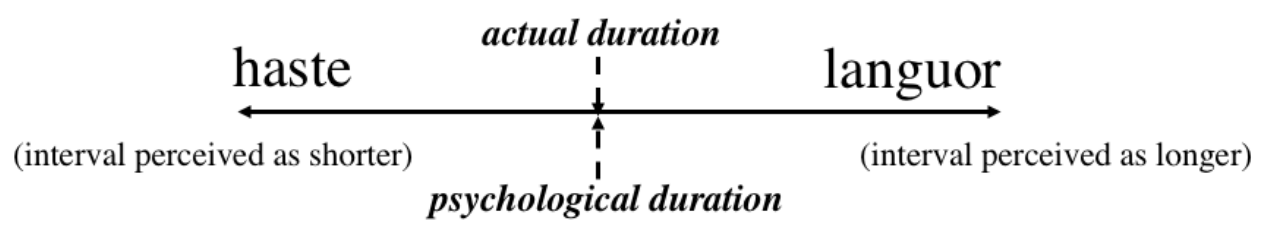

Figure 1. Psychological Time Continuum

Absolute time represents objective durations which rarely represent subjective durations perceived by separate individuals. For example, a week to a young child seems to last a lot longer than to a middle-aged person. There are many potential explanations for the differences in duration judgements involving various ages, or indeed different people of the same age. Among the proposed reasons that include both biological and cognitive processes, are: the changing of metabolic rate, brain temperature, internal pacemaker rate, psychological factors, new experiences, the amount of information being processed and the speed of processing, the person's allocation of attentional resources, keeping oneself busy or idle, impatience and 
waiting, etc. (This information is based on various sources; many experiments on the subject are summarised and analysed in Block, Hancock and Zakay 1999.) For the above reasons, the central point of the psychological time continuum is hardly ever reached at the same time by all members of an audience, even when it appears that they share neutral feelings of time passing.

\section{FACTORS INFLUENCING TIME PERCEPTION}

Perception of durations and time passing is influenced by several factors that emerge from music, belong to a listening environment, or exist as inherent conditions of the listener. I arrived to this set of factors through my research mainly on cognitive psychology, observations and experiments I conducted through my compositions. 2 Figure 2 shows the combination of these factors, divided into two main categories, those directly related to music and those that originate outside it.

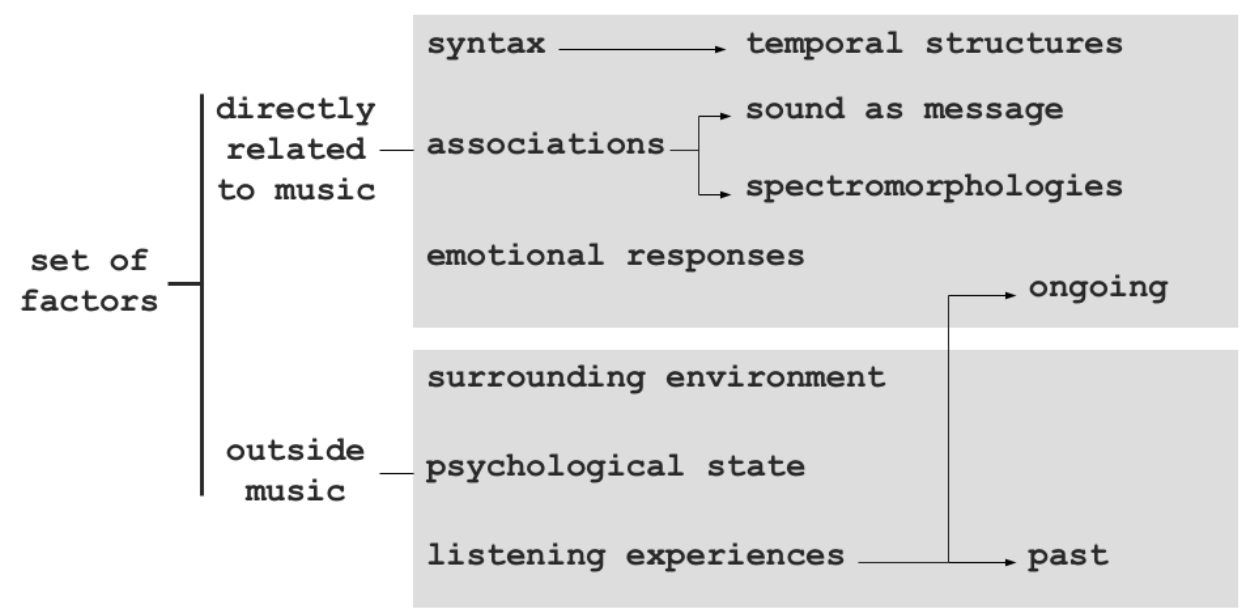

Figure 2. Set of Factors

Factors related to music are the temporal structures, temporal associations of sounds carried through their semantic meanings and also through their spectromorphological characteristics, and the emotional responses of the subject to 2 Details of my research can be found in my unpublished $\mathrm{PhD}$ thesis (Pasoulas 2011c). 
the object of perception. Associations as an influencing factor have been explored in Pasoulas 2011b, where extra-musical links and spectromorphologies are discussed in detail; gestural and textural material may relate to motion unfolded in a variety of source-causes (linked to the real world or implied through spectromorphological content), displaying various degrees of urgency, i.e. a position within the haste/languor continuum. The factors originating outside music comprise the surrounding environment at the time of listening (which includes the aural, visual, haptic, gustatory and olfactory environments), and the psychological state of the listener. In this diagram, listening experiences are divided into two kinds. Past experiences originate outside ongoing music listening, arising from cultural tendencies, education and autobiographical experience. Ongoing cumulative listening experience is directly related to music because syntax and messages as well as emotional responses evolve with renewed meaning (depending on the analytic competence of the listener), formed by the accumulated experience of their context. Some of the factors in Figure 2 can be further expanded and include supplementary factors. In the case of ongoing listening experiences, expectation is emerging as a supplementary factor; and in the case of temporal structures, an auxiliary factor is complexity, which may appear in several forms: rhythmic and melodic, spectromorphological and spatiomorphological complexities, referential density, referential discourse density and harmonic complexity (discussed in detail in Pasoulas 2011a).

This combination of factors can be regarded as a group of filters that colour the temporal information of a musical piece. Because the values of these filters are unique for certain groups of people, or indeed for each person, subjective durations perceived by separate individuals may differ to various degrees. Each factor requires detailed 
discussion which cannot be accomplished in one paper. The rest of this paper concentrates on the influence of our listening experiences, psychological states and emotional responses to our perception of time, and considers the idiosyncratic performance of the proposed group of filters.

\subsection{Listening Experiences}

The accumulation of listening experiences is a process that operates in two ways: as a long-term action that depends on place, era, and individual learning activities, and as an ongoing cognitive activity that allows new sonic experiences to be absorbed and used in the continuing process of listening. The first operation can be regarded as forming part of the intangible cultural heritage 3 of the listener, because the same sonic experiences that define a place and an era are shared by the listener's community. However, individuals develop additional repertoires of sound experiences, depending on their activities.

Past listening experiences are largely responsible for the interpretation of acoustic environments and sounds. As shown in Pasoulas 2011b, both semantic suggestions and spectromorphological attributes of a sound can relate to extra-musical references, and their interpretation depends on the upbringing and the accumulated experiences of a person. Foghorns mean nothing to people who come from countries where there are no harbours (or no fog), and bells of old London routemaster buses are unknown to people who never had that listening experience. When listening to a piece of music, our experience of hearing events in everyday life compels us to interpret semantic references and morphological characteristics into representational images, based on

3 According to UNESCO convention, intangible cultural heritage consists of non-physical characteristics and knowledge that define a cultural group. Among others, it includes oral traditions and social practices, performing arts and rituals, festive events, and knowledge and practices concerning nature. It is transmitted through generations, and is renewed by communities in response to changes in their environment (UNESCO n.d.). 
our acquired knowledge. Sounds, especially those related to action, may be associated with various levels of haste or languor, such as the sound of an airplane taking off, or a swift gesture of a sword in a video game. Sounds set up contexts and define places and times.

Cultural tendencies are not borne only by geographical associations and group or individual activities, but also by associations with particular historical periods. There are sounds disappearing from large areas, thus rendering certain sonic environments obsolete (Truax 1999); those sounds make sense only to generations that have experienced them at certain periods and places, e.g. air strike alarm. Air strike alarm may induce a higher degree of urgency to someone who has experienced it in real life, compared to others who have not. Memories of past listening experiences and their interpretation are present even before the music is heard, which means that certain sounds are preconditioned by the listener to be perceived in a certain way.

However, new listening experiences are continuously absorbed as a cumulative process both in everyday life activities and while music is heard, which allows for these new experiences to engage in the interpretation of the sonic material. The analytic competence of the listener, which arises from the educational background and previous experiences, influences the interpretation of material, and selects convenient messages (temporal or otherwise), favoured by the listener's experience.

A good example of the influence past listening experiences have on our interpretation of time information is the sound cicadas and crickets make; they both imply a specific temporal setting, i.e. day and night respectively, in the hot months of the year, because they appear during those periods. If those sounds are experienced in a recording, the time and season associated with them can only be interpreted correctly by people who are familiar with the sound of both insects in their respective 
environments. Day and night usually associate with busy state and calmness respectively, which relate to haste and languor in the psychological time continuum. 4

\subsection{Psychological States}

The psychological state of the individual at the time of listening affects the way that time passing in music is perceived. Mental and physical tiredness, as well as anger, happiness and sadness influence the time perception of the listener, inducing a state where a person becomes agitated, relaxed, or inundated with information (in the case of mental tiredness), resulting in a distorted judgement of durations and time passing.

Psychologist Allessandro Angrilli and his colleagues cite several studies from 1960 onwards (1997: 973) offering evidence that both positive and negative emotions affect time perception. Angrilli based his own experiments on the dimensional analysis of emotions, which is a leading theoretical approach to analysing emotional states. Basic emotion theories are based on the presence or absence of emotional states, whereas dimensional theory considers a multi-dimensional space, where various factors that affect emotions vary along a continuum (Scherer 2004: 246-8). Angrilli claims that there are two main factors responsible for most of the variation in emotional judgements; affective valence (that corresponds to direction, i.e. attraction or repulsion that correlate with positive or negative reaction to a stimulus) and arousal (corresponding to intensity of the emotional state) (op. cit.: 972). His results demonstrate that there are two different mechanisms for duration estimation, one activated at low arousal levels and the other at high ones, which generate opposite responses at positive and negative affective valence. At low arousal situations,

\footnotetext{
4 My acousmatic composition Paramnesia explores day and night and their associated activity and temporal settings. It is published by ICMA (ICMC2010 CD) and can also be found online (https://soundcloud.com/aki_p/paramnesia). Detailed notes on the composition are in my unpublished $\mathrm{PhD}$ thesis (Pasoulas 2011c: 123-30).
} 
positive valence induces overestimation of durations, while negative causes underestimation. At high arousal situations the behaviour is opposite; positive affective valence leads to underestimation, while negative to overestimation of durations. Angrilli maintains that when the arousal level is low, the mechanism is attention-driven, and when it is high, the mechanism is emotion-driven. He claims that the dual mechanism is in accordance with a biologically adaptive function, because the attention-driven mechanism optimizes the collection of relevant information from the environment during low information conditions, while the emotion-driven mechanism facilitates the reaction to possibly dangerous or gratifying situations, without delay (ibid: 980). Among the studies cited by Angrilli are Watts and Sharrock's experiment in 1984, which demonstrated that spider-phobic individuals overestimate durations when watching spiders; and the study by Langer, Wapner and Werner in 1961, which showed that people overestimate durations when looking at unpleasant (e.g. angry) facial expressions.

Although Angrilli's experiments were conducted with visual stimuli which were used to induce emotional states, his evaluation is focused on the relationship between emotions and psychological durations - the nature of the stimuli becomes irrelevant. Hence, his observations can apply to many contexts, including listening situations, where emotions affect duration estimation in different and sometimes contrasting ways; for example, members of an audience may have a predisposition to underestimate durations due to their current low intensity negative emotions, compared to overestimated durations experienced by other members of the same audience with low intensity positive emotions.

Gorn, Chattopadhyay, Sengupta and Tripathi (2004) offer alternative explanations for the overestimation of durations under stressful situations. (1) Under pressure or 
emotional stress, the internal clock5 accelerates, so that more time is experienced through that clock than it is in reality, which results in a perception of time passing slowly; and (2) people in general wish for unpleasant situations to pass quickly, and so the anticipation created makes it seem that time drags (ibid: 216). The second suggestion clearly relates to the experience that listeners have when they do not like the music they hear.

Hawkins, French, Crawford and Enzle (1988) measured the subjective time passing on depressed people and concluded that time for them passes more slowly than for people on neutral and elated states. Mental illnesses that alter the psychological state of patients, as well as psychoactive drugs such as marijuana and cocaine, slow down and speed up time perception respectively. Music theorist Jonathan Kramer cites psychiatrist Roland Fischer who claims that drugs arouse symptoms already 'present within the cerebral organisation' (1988: 381). Kramer's point is that experiences of slowing down or speeding up auditory images are available to the human mind and so it is possible to be induced.

Personality traits can also influence time judgments. According to psychologist Wayne Hogan, extroverts need and tolerate more stimulus input than the introverts, because their sensory threshold is higher. Consequently, extroverts experience time filled with least degree of complexity as longer than the same interval experienced by introverts (Hogan 1978: 420, 423-4).

\subsection{Emotional Responses}

5 The hypothetical internal clock is discussed in many writings, since at least the 1920s. Two early contributions on the subject can be found in Francois M. (1927) and Hoagland, H. (1935). In brief, the most prevalent timing theory today, the scalar timing theory, assumes that psychological time is produced by an internal pacemaker that emits pulses at some rate which follows the pace and changes of external stimuli (Wearden 1991: 60). 
Another factor that influences the perception of musical time is the emotional responses of the listener to sounds. Denis Smalley, based on Ernest Schachtel's autocentric perceptual mode, discusses the 'reflexive relationship' between the listener and sounds, stating that in this subject-centred relation 'the object has no real identity separate from the subject's emotion' (Smalley 1996: 82). He maintains that in this particular case, there is little or no exploration of the sound material, only subjective responses of enjoyment or displeasure. These emotional responses to sound material can induce certain psychological states manifesting attraction or aversion in various intensities, which alter the perception of time passing (as explained above in section 3.2).

David Huron (2005) suggests that evolutionary adaptations may influence musical experience through our emotional reaction to sounds. From his suggestion, we can deduce that biological trends may become compositional tools with which composers can influence emotional responses and, by extension, the experience of time passing and perception of durations. Huron illustrates his argument by referring to examples that evoke positive feelings that accompany parenting behaviours. For his experiments, he used sounds that displayed 'auditory cuteness' (ibid: 6), such as little bells, small animal calls and squeeze toys, all of which displayed high pitch, high spectral centroid and low amplitude. Huron maintains that the experience of the characteristics of 'auditory cuteness' stimulate positive feelings and 'a distinctive psychological state that includes nurturing and protective behaviors' (ibid). He affirms that cuteness is not an objective property of sounds, but a 'disposition of the mind designed to enhance adaptive fitness' (ibid: 6). It is possible that other types of sound material can evoke certain negative or positive primary feelings, based not on the recognisability of the sound source, but on the spectromorphological attributes of 
the sounds. Spatial position can also influence emotional responses, as noted by Wishart (1996: 199-200). Frightening sounds (e.g. low frequency loud impacts) heard from behind are more startling, threatening or stressful than if they were heard from the front.

François Delalande (1998) examines reception behaviours and encounters three main types that can be combined dynamically during listening; taxonomic listening behaviour (concerned with structure), empathic listening (related to experienced sensations), and figurativisation (where certain sounds are likened to living beings). Delalande emphasises that these are not the only listening behaviours, noting that 'listening adapts itself to the object' (ibid: 59). He also notes that the resolution of a conflict between two behaviours, as well as the changing of listening strategies during audition (possibly because the behaviour of sounds changes and the adopted strategy does not work anymore) result in 'observable emotional responses' (ibid: 63). Considering the research cited previously on the effect of psychological states on duration estimation (section 3.2), it is very likely that these emotional responses partly account for variations in perceived structural balance of the same piece of music among listeners who may follow different combinations of listening strategies. Moreover, the emotional responses due to changing of listening strategies in the midst of audition may partly explain why we sometimes perceive disparate sections that otherwise have similar physical durations as extremely unbalanced. The composer has to work on psychological durations to achieve the desired proportions, especially when the behaviour of the sound material changes dramatically during the piece; acquiring the right balance can be problematic considering that people may adopt different listening strategies. In concerts, it may be possible to anticipate certain listening behaviours shared among listeners by targeting specific audiences, for 
example by organising concerts that have specific themes (e.g. environmental sound), or events in controlled listening environments (e.g. thematic sound installations in a gallery). That would at least ensure that audiences are likely to follow similar listening strategies, which may reduce wide deviations in time perception among listeners.

With regard to empathic listening, Delalande reports that listeners describe various gestures 'not as if they have witnessed a scene from a distance but rather as if they have been subjected more or less to these movements themselves' (ibid: 37). His subjects point out that they almost feel the gestures physically. It is interesting that they experience the piece chosen by Delalande (Pierre Henry's Sommeil, composed in 1963) in the present moment, without trying to establish relationships with past material (ibid: 38). While listening to the piece, his subjects described sensations as physiological products of the sounds; stomach-blow impact, weightlessness, heaviness, thickness, rubbing sensation etc. Sometimes, they used complex descriptions of narrative images that clarified their own reactions; for example, 'karate in slow motion' and 'blows thrust at you by a wooden sabre' (ibid: 38-9). Delalande admits that one cannot know whether such images were present during listening or they were fabricated later to verbalise the felt sensations. However, what is important for our argument is not the presence or the absence of an image, but the felt sensation.

It is understood that physiological arousal provides a necessary basis for sensation; e.g. an external stimulus such as low atmospheric temperature generates a nerve discharge from the (involuntary) sympathetic nervous system, which causes a contraction of hair erector muscles that elevate the hair follicles above the skin, and we experience piloerection or gooseflesh. Psychological research shows that emotions 
are often associated with reaction to such sensory information (de Sousa 2007). The most important theories on the relation between physiological arousal and emotion have been developed by William James and Carl Lange in 1884 (arousal leads to emotion; Cannon 1927), Walter Cannon and Philip Bard in 1927 (emotion occurs at the same time as the physical response; ibid.), and Stanley Schachter and Jerome Singer in 1962 (two-factor theory: physiological arousal and its cognitive label lead to emotion; Reisenzein 1983). Considering the connection among sensation, somatic experience and emotion as explored in the above main theories, it is reasonable to assume that the felt sensations of the listeners (as described by Delalande) are frequently - if not always - connected with certain emotional responses, which can affect the perception of time.

However, sensations may not connect to similar emotions when experienced by different persons; for example, weightlessness may be associated with euphoria for one person, but with fear for another. Nevertheless, the emotional judgements (derived from the combination of affective valence and arousal) will influence the perceived durations of sound events. Ultimately, time perception will leave a certain impression about the pace and momentum of the piece of music. Delalande points out that empathic listening does not favour the perception of sections (1998: 41), which implies that, although pace may be affected, the overall structural balance will not necessarily be influenced by the changing time perception due to emotion. In other words, in empathic listening, time passing and pace (related to the experience of the present) are affected, while duration estimation (related to remembered durations) is not.6 
It is also important at this point to give some relevant information related to Delalande's notes on the perception of stasis. Delalande mistakenly identifies the fixation with the present as stasis (1998: 38), which does not agree with the continuous process of change or flowing movement experienced by his subjects. Moreover, Delalande believes that 'the piece is perceived as an instant' (ibid: 42). According to previous research in psychology (James 1890; and Pöppel 1978), the time interval in which information is experienced as a single unit is generally accepted to be c.5 sec long, and it is called 'specious present' or 'subjective present'; it is organised as an oscillatory process that follows excitation and relaxation modes. However, when Delalande's article later examines form perception (1998: 45), he comes close to admitting the presence of an interval surrounding that instant; it is this extra duration around the strict present that forms the specious present.

An interesting characteristic of empathic listening behaviour is that, because sounds are interpreted as movements related to the body of the listener (i.e. the space is given), the temporal scale of the gestures is interpreted accordingly. According to Delalande, since human movement of feet, head or arms (at normal speed) does not generally exceed the duration of one second, longer gestures are perceived as extremely slow (ibid: 47). The impression of slow and fast motion can be extended to listeners that do not necessarily apply empathic listening, by deliberately using sounds that create certain speed expectations when resemble those of body movements. 7 An extreme example can be considered in multi-channel surround sound, when a series of short gestures that display spectromorphological characteristics of footsteps is moved in a circle around the listener. The speed of execution of the circular motion determines the perception of distance between two steps of the virtual walker; the 
normal speed is dictated by the distance between successive loudspeakers. Very slow speeds make the virtual person seem as if s/he steps too many times around the same spot, whereas high speeds result into huge steps in space; anything beyond the normal speed sounds unnatural, and it takes practice and patience to adjust the spatial motion to the pace of the walk.8

\section{THE TIME-INFLUENCING SET}

Although much of this research has a broader scope and is relevant to music and sound art in general, my main focus as a composer is on electroacoustic music and more specifically on acousmatic. When acousmatic music is seen from an ecological perspective, it can be recognised that there are interrelationships between music, the listener and the listening environment. As a result of this observation, the impact that listening environments, and state and experience of listeners have on the perception of musical works should be questioned and carefully considered. Although acousmatic music may appear to invite a pure listening experience, that is, not directly involving other senses, it has a more holistic nature. Acousmatic music incorporates, mostly unintentionally, all its surrounding elements at the time of listening.

Our perception of duration and time passing changes according to a mixture of numerous and sometimes disparate elements. The combination of these elements, which are the factors shown earlier in Figure 2, produces certain results in the psychological domain. Together, these factors may collectively be regarded as a 'time-influencing set' (referred from now on as the T-I set), where 'set' is a collection of distinct factors, each one serving the same purpose in this case, i.e. to influence the

8 I regularly use this demonstration in my classes on multi-channel composition to show the consequences of motion and speed expectation created by the associations of sounds. The influence that this simple demonstration has on students' thinking about gesture and space is profound. 
time perception of the listener. The process whereby the T-I set influences listening is shown in Figure 3. The T-I set is placed between the listener and the music (EA) and acts as a filter, which is in constant flux, and which shapes the time perception of the piece. It will be made clear later why this filter is in flux.

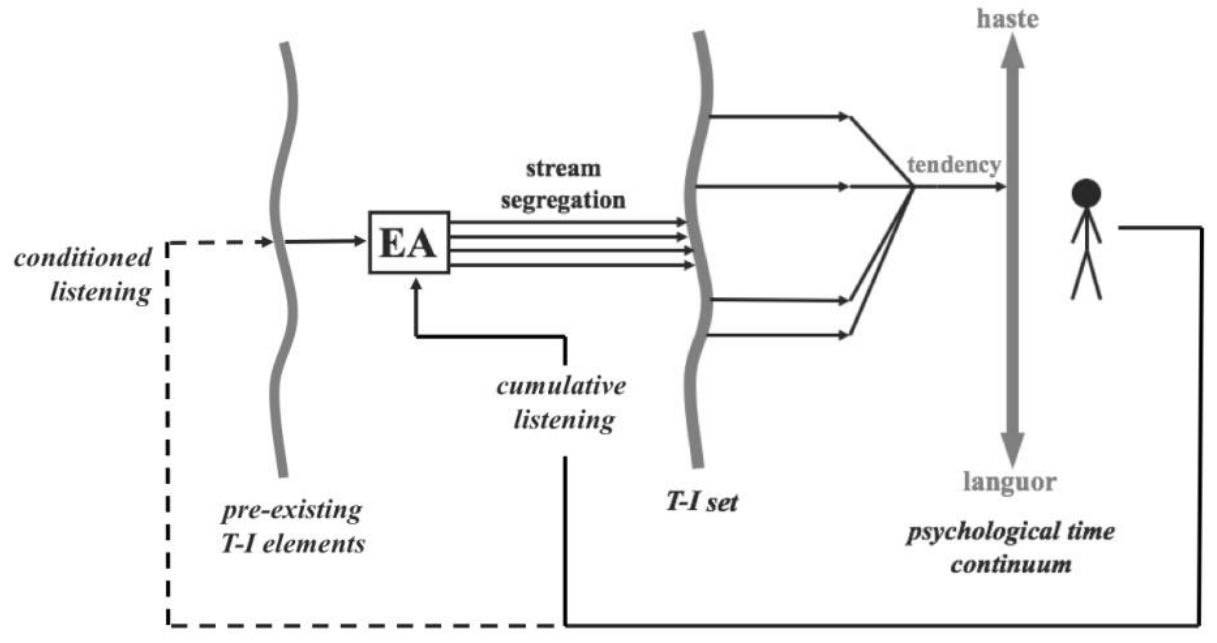

Figure 3. The Time-Influencing (T-I) set and its relation to the music and the listener.

Some T-I elements are already present even before the music is heard, because they constitute part of the individual person, i.e. the psychological state of the listener and past listening experiences ('pre-existing T-I elements' in the above diagram). This means that auditory streams emerge out of the music already filtered, which implies that every individual is conditioned to perceive the timescales of a piece of music in a certain way, even before they emanate from their source. This of course is true not only for electroacoustic music, but for any type of sound structures. Then, further filtering will occur due to factors that shape the music, and which constitute the main body of the T-I set. Ongoing cumulative listening experience is indicated by the feedback arrow that points to music.

The psychological time continuum is positioned graphically close to the listener, although it is inseparable from the person; different locations on this continuum 
correspond to subjective experiences of periods of time in which sequences of sound events take place. Sounds are grouped into auditory streams according to their characteristics (see stream segregation9 in Figure 3) and they pass through the T-I set, each stream occupying a timescale; streams may also interact with each other, thus forming composite, discrete timescales.

A short parenthesis with a sound excerpt taken from my composition Chronos (2006) can demonstrate a case of discrete timescales (Sound example 3). The excerpt is represented graphically in Figure 4. A composite timescale dissolves into fission of granulated textures and discloses three discrete decelerating streams of micro events, which morph into ticking clocks running at different rates. The curves in the timescale diagram $(\mathrm{R})$ do not relate to the curves of the sonogram $(\mathrm{L})$, but rather to the transients which are visible, and can be seen elongating towards the end of the sonogram. The light grey line in the diagram area corresponds to the fluid, nondiscrete motion of the low frequency texture heard in the sound example, which has been omitted in the sonogram for the sake of clarity. The three timescales display different rates of time passing, and they form three superimposed oscillating systems.

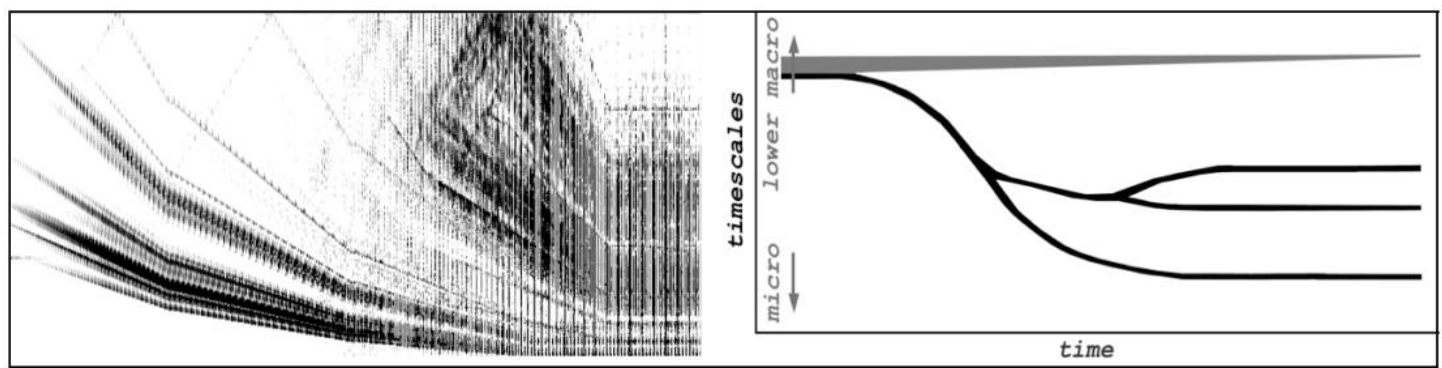

Figure 4. Decelerating clocks; sonogram analysis (L) and timescale diagram (R).

9 Bregman identifies auditory stream segregation as the process in which “... links are formed between parts of sensory data ... [which] affect what is included and excluded from our perceptual descriptions of distinct auditory events" (1999: 47). 
We now go back to the diagram in Figure 3. After the auditory streams pass through the T-I filter, they acquire new values in terms of time perception. What we sense then, is a point of inclination, a tendency towards a qualitative value on the psychological time continuum that arises from the interaction among the filtered auditory streams. The process of entrainment can explain the merging tendency of the different streams, as these constitute oscillating systems that our hypothetical internal pacemaker attempts to bring together. Consequently, the slow oscillating system accelerates perceptually, whereas the fast one decelerates; the degree of acceleration/deceleration depends on the strength of each system and on how far apart these systems are. The listener can experience a change in pace of sound information belonging to the focal field, whereas the secondary oscillators remain unnoticed. The entrainment process, in relation to listener's attention, is analysed in Jones 2004. The hypothetical internal pacemaker is discussed in many writings, since at least the 1920s. Two early contributions on the subject can be found in François (1927) and Hoagland (1935). In brief, a prevalent timing theory today, the scalar timing theory, assumes that psychological time is produced by an internal pacemaker that emits pulses at some rate which follows the pace and changes of external stimuli (Wearden 1991).

When the T-I set is analysed into its constituent parts (sound as message, aural, visual environment, etc.), it can be speculated that auditory streams change their direction individually, pointing towards either haste or languor, while passing through the parameters of this multiple filter. The tendency point gives us a location on the psychological time continuum (Figure 3), a qualitative measure for perceptual duration. 
The T-I set is in constant flux because it is highly unlikely that all individual continua will stay fixed during a performance of a music piece; some will be unchanged, like the performance space (which is part of the surrounding environment), but will not be able to suspend the fluctuation of the tendency point. When the listener focuses on a particular auditory stream, then that stream has a stronger influence on the tendency point. Moreover, when a value in the T-I set changes (e.g. when introducing a sound that carries a new temporal connotation), the tendency point will change its position accordingly.

The T-I set works differently in cases where the listener can clearly distinguish several rates of time passing (as in the last few seconds of Sound example 3). Perceptual segregation of timescales can be facilitated by cues used by the auditory system to differentiate among simultaneous sound images. Such cues include spatial position, common fate regularity (where several partials evolve in a similar way), partial onset (since the ear tends to group together partials starting at the same time), and the use of harmonic series (a harmonic series will probably derive from the same source) (Bregman 1999). For example, when timescales are clearly separated in the panoramic space, the listener is able to segregate the temporal streams and shift attention among them. During spatial segregation, timescales with very different pace will have their tendency points on the psychological time continuum move apart towards the opposite poles (haste/languor); consequently, the attention of the listener will shift between the qualitative values depending on the area of focus. Listeners probably cannot feel two rates of motion at once (haste and languor), but are nevertheless aware of the existence of both because they can switch between them.

There is a perceptual threshold beyond which streams of temporal information carried by auditory streams are not merged. That threshold depends on the shared 
characteristic that brings the group together; for example, if the shared characteristic is the spatial position of the sounds, the threshold depends on spatial distance; if it is frequency, it depends on the distance between frequency bands, etc. Streaming in terms of time perception seems to work similarly to auditory streaming, that is, temporal streams are also organised perceptually according to their similarities. However, auditory streaming and temporal streaming are not the same thing; a number of segregated auditory streams may operate on the same timescale, since they can move forwards at the same pace and occupy the same period of time. Similarly to auditory streaming, there are several parameters in temporal streams that either compete or collaborate in order to determine grouping. These parameters are to be found in temporal syntax and associations, which establish temporal relationships grouping - among sound images. 10

\section{SUMMARY}

In this paper, it has been suggested that there are two distinct ways of experiencing time, the first through the notion of absolute time, and the second through psychological time. The latter is ultimately connected with our understanding of a musical piece. Absolute time can be divided into several timescales; a modified division of Roads' timescales gives us four durations that make sense to listeners: lower macro, meso, sound object and micro. However, psychological time cannot be partitioned in the same way. A psychological time continuum has been proposed, where different positions represent differing perceptions of physical durations.

The listener is conditioned through personalised factors to perceive timescales in a certain way, even before these emerge from music. Past listening experiences 
influence the interpretation of sounds, acoustic environments and temporal messages, whereas absorbing new listening experiences while music is heard allows for a cumulative process to take place resulting in a renewed insight of sonic material.

The psychological state as well as personality traits of the listener affect the way that time passing and durations are perceived. Moreover, time judgments are influenced by the emotional reaction of the listener to sounds. Our perception of time changes according to a mixture of elements that make up the Time-Influencing (T-I) set. The T-I set operates as a filter, producing certain results in the psychological domain which affect the listener's time judgments.

\section{REFERENCES}

Angrilli, A., Cherubini, P., Pavese, A. and Manfredini S. 1997. The Influence of Affective Factors on Time Perception. Perception and Psychophysics 59 (6): 97282.

Block, R. A., Hancock, P. A. and Zakay, D. 1999. Developmental Changes in Human Duration Judgments: A Meta-Analytic Review. Developmental Review 19: 183211.

Bregman, A. S. 1999. Auditory Scene Analysis: The Perceptual Organization of Sound. 2nd edn. Cambridge, Massachusetts: MIT Press.

Cannon, W. B. 1927. The James-Lange theory of emotion: A critical examination and an alternative theory. American Journal of Psychology 39: 106-124.

Delalande, F. 1998. Music Analysis and Reception Behaviours: Sommeil by Pierre Henry. Journal of New Music Research 27 (1-2): 13-66.

de Sousa, R. 2018. Emotion. The Stanford Encyclopedia of Philosophy. http://plato.stanford.edu/entries/emotion (accessed 16 August 2019). 
François M. 1927. Contribution à l'étude du sens du temps. La témperature interne comme facteur de variation de l'appréciation subjective des durées. L'Année Psychologique 27: 186-204.

Gorn, G. J., Chattopadhyay, A., Sengupta, J. and Tripathi, S. 2004. Waiting for the Web: How Screen Color Affects Time Perception. Journal of Marketing Research 41: 215-25.

Hawkins, W. L., French, L. C., Crawford, B. D. and Enzle, M. E. 1988. Depressed Affect and Time Perception. Journal of Abnormal Psychology 97 (3): 275-80.

Hoagland, H. 1935. Pacemakers in Relation to Aspects of Behavior. Oxford: Macmillan.

Hogan, H. W. 1978. A Theoretical Reconciliation of Competing Views of Time Perception. The American Journal of Psychology 91 (3): 417-28.

Huron, D. 2005. The Plural Pleasures of Music. Proceedings of the 2004 Music and Science Conference. Stockholm: Kungliga Musikhögskolan and KTH Royal Institute of Technology, 1-13.

James, W. 1890. The Principles of Psychology, Vol. 1. New York: Henry Holt. Jones, M. R. 2004. Attention and Timing. In J. G. Neuhoff (ed.) Ecological Psychoacoustics. California and London: Elsevier Academic Press, 49-85.

Kramer, J. D. 1988. The Time of Music: New Meanings, New Temporalities, New Listening Strategies. London: Collier Macmillan.

Pasoulas, A. 2001. Stockhausen and Formel Komposition. NoiseGate 9: 50-6. Pasoulas, A. 2011a. Stimulus Complexity and Time Judgments. Proceedings of the International Computer Music Conference, 163-5.

Pasoulas A. 2011b. Temporal Associations, Semantic Content and Source Bonding. Organised Sound 16(1): 63-8. 
Pasoulas A. 2011c. The Perception of Timescales in Electroacoustic Music.

Unpublished $\mathrm{PhD}$ thesis. http://openaccess.city.ac.uk/id/eprint/1155/ (accessed 20 August 2019).

Pöppel, E. 1978. Time Perception. The Handbook of Sensory Physiology VIII: Perception. Berlin: Springer-Verlag.

Reisenzein, R. 1983. The Schacher Theory of Emotion: Two Decades Later. Psychological Bulletin 94 (2): 239-64.

Roads, C. 2001. Microsound. Cambridge, Massachusetts: MIT Press.

Scherer, K. R. 2004. Which Emotions Can be Induced by Music? What Are the Underlying Mechanisms? And How Can We Measure Them? Journal of New Music Research 33 (3): 239-51.

Smalley, D. 1996. The Listening Imagination: Listening in the Electroacoustic Era. Contemporary Music Review 13 (2): 77-107.

Truax, B. 1999. Disappearing Sound. Handbook for Acoustic Ecology. Cambridge Street Publishing. http://www.sfu.ca/sonic-studiowebdav/handbook/Disappearing_Sound.html (accessed 10 August 2019).

UNESCO. n.d. What is Intangible Cultural Heritage? https://ich.unesco.org/en/whatis-intangible-heritage-00003 (accessed 14.08.2019).

Wearden, J. H. 1991. Do Humans Possess an Internal Clock with Scalar Timing Properties? Learning and Motivation 22: 59-83. 